\title{
Genome organization via loop extrusion, insights from polymer physics models
}

\author{
Surya K. Ghosh ${ }^{1,2}$ and Daniel Jost ${ }^{1,3, *}$ \\ ${ }^{1}$ Univ Grenoble Alpes, CNRS, Grenoble INP, TIMC-IMAG, F-38000 Grenoble, France \\ ${ }^{2}$ Department of Physics and Nanotechnology, SRM Institute of Science and Technology, \\ Kattankulathur, 603203, Tamil Nadu, India \\ ${ }^{3}$ Laboratory of Biology and Modelling of the Cell, Univ Lyon, ENS de Lyon, Univ Claude Bernard, \\ CNRS UMR 5239, Inserm U1210, F-69007 Lyon, France \\ ${ }^{*}$ Corresponding author: daniel.jost@ens-Iyon.fr, +334 72728000
}

\begin{abstract}
Understanding how genomes fold and organize is one of the main challenges in modern biology. Recent high-throughput techniques like $\mathrm{Hi}-\mathrm{C}$, in combination with cutting-edge polymer physics models, have provided access to precise information on 3D chromosome folding to decipher the mechanisms driving such multi-scale organization. In particular, Structural Maintenance of Chromosomes (SMC) proteins play an important role in the local structuration of chromatin, putatively via a loop extrusion process. Here, we review the different polymer physics models that investigate the role of SMCs in the formation of topologically-associated domains (TADs) during interphase via the formation of dynamic loops. We describe the main physical ingredients, compare them and discuss their relevance against experimental observations.
\end{abstract}

\section{Keywords}

Chromosome organization; modeling; polymer; loop extrusion; SMC proteins

\section{Summary key points}

- SMC-mediated chromatin loops may be formed via a loop extrusion process.

- Polymer physics models have been instrumental in studying loop extrusion.

- Loop extrusion may be driven by: directed translocation of active motors, diffusion of slip-links, transcription-induced supercoiling.

- We describe the main physical ingredients and parameters to better understand and compare these three classes of models.

\section{Introduction}

Inside cellular nuclei, genomic DNA is tightly packed into a polymer-like structure called chromatin whose local conformation modulates the accessibility and specificity of regulators to the DNA sequence [1]. Until recently, chromatin was mainly studied as a unidimensional object and little attention was paid to its three-dimensional structure. In the last decade, thanks to the development of chromosome conformation capture ( $\mathrm{Hi}-\mathrm{C}$ ) and advanced microscopy techniques, major progress has been realized in our understanding of 
chromosome organization during interphase $[2,3,4]$. The genome is locally partitioned into conserved $\mathrm{kbp}$ - to $\mathrm{Mbp}$-size domains with a relatively high number of contacts, known as topologically-associating domains (TADs) that usually encompass promoter-enhancer looping interactions. At larger-scale (1-10 Mbp), loci with similar gene activity or epigenomic content self-organize into spatial compartments [5-8]. Finally, at the nuclear level, chromosomes occupy separate 3D territories that only weakly intermingle $[9,10]$ and functional chromatin compartments preferentially localize at the periphery for heterochromatic regions or centrally for euchromatic segments [11, 12]. This peculiar multi-scale organization is observed in most of higher eukaryotes, from drosophila to worm and mammals [13], suggesting that 3D organization plays a fundamental role in the regulation of gene expression. Indeed, many studies have illustrated the role of TAD and TAD boundaries in promoting or preventing enhancer-promoter interactions for important genes during development or diseases [1420], even if several recent works tend to question a global, direct effect of the local 3D organization on transcription [7, 21-23].

While the description of 3D chromatin folding is becoming more and more quantitative, our understanding of the molecular mechanisms that drive the 3D genome organization remains sparse. Several processes have been shown or suggested to play major roles in organizing chromosomes [24]. Formation of epigenomic or other nuclear, membrane-less compartments is putatively mediated by chromatin-binding proteins that have the capacity to self-interact [25-27] and potentially phase-separate with the formation of protein micro-droplets where specific chromatin segments may colocalize [28-30]. TAD formation in mammals has been associated with the translocation along the genome of SMC complexes, like condensin or cohesin rings, and with their association with insulator proteins bound at TAD boundaries, like CTCF [31-34]. In this review, we will focus on this latter family of mechanisms, which are often referred to as loop extrusion processes in the literature.

Application of the loop extrusion process to explain TAD formation originated from the combination of recent high-resolution $\mathrm{Hi}-\mathrm{C}$ experiments on interphasic mammalian nuclei with older concepts originally developed for the condensation of mitotic chromosomes. In recent years, improvements in $\mathrm{Hi}-\mathrm{C}$ resolution allowed to get a more precise description of TAD organization and substructures. In particular, in mammals, several groups observed that many TADs possess an enrichment of contact between the two TAD boundaries leading to corner-peaks in Hi-C maps [7] (Fig.1A) or between a boundary and the interior of the TAD leading to prominent stripes [49] (Fig.1B). Careful analyses of these peak signals showed that contacts occur mainly between convergent CTCF-binding sites usually located at domain boundaries $[7,32,35,36]$ and are associated with the presence of cohesin SMC complexes (Fig.1A, B). The preferential sense-antisense orientations between contacting loci cannot be simply explained by specific, 3D interactions [32] (Fig.2A) but are instead consistent with a 1D mechanism of loop formation along the genome (Fig.2B,C). Actually, this type of process was firstly hypothesized by Riggs [110] and Nasmyth [38] to explain the formation of 1D arrays of contiguous loops in mitotic chromosomes (Fig.2B) by condensin SMC complexes, and was clearly formalized by Alipour and Marko [39] in 2012 and coined loop extrusion. In 2015, based on these ideas, Lieberman-Aiden's and Mirny's groups concurrently suggested that TAD formation followed the same basic principle: loop extruding factors, likely to be cohesin rings in mammals, are loaded onto chromatin (by Nipbl proteins), sequentially extrude large chromatin loops, and eventually dissociate from DNA (thanks to Wapl proteins) or are halted as they encounter boundary elements (CTCF-bound motifs) with specific orientation (Fig.2C). While the molecular details of the loop extrusion mechanism remain unclear, many direct in 
vitro observations have confirmed that the ATP-driven activity of SMCs may induce DNA $[37,67,111]$ or chromatin [100] compaction, and that condensin exhibits a loop extrusion-like activity on naked DNA $[40,41]$. Moreover, in vivo, loop extrusion is supported by many indirect experiments in which, for example, the levels of cohesin (or of Nipl or Wapl) and of CTCF were manipulated, or where the positions or orientations of CTCF motifs were modified [31, 42-49] (see [34] for a detailed review on these evidences).

Beyond TAD formation in mammals or mitotic condensation in eukaryotes, loop extrusion mechanism by SMCs has been involved in the regulation of many fundamental biological processes like the meiotic chromosome assembly in yeast [112] or the juxtaposition of chromosome arms in bacteria [106,113]. It is therefore of crucial importance to better characterize this process. In recent years, polymer models have been instrumental in showing that the loop extrusion mechanism was consistent with experimental data. In this article, we review, compare and discuss the different polymer models that have been developed to describe and explain the loop extrusion process. In particular, we aim at describing the physical ingredients, the main parameters, and their typical values for a non-expert reader to better understand the physical grounds of these models and their relative differences.

\section{Polymer physics models of loop extrusion}

One of the great advantages of mechanistic or bottom-up modeling in biology is the possibility to test, based on first principles, if putative mechanisms, processes or hypotheses are compatible with experimental observations, while direct validation might be experimentally difficult due, for example, to technical limitations or because the involved actors have ubiquitous functions. Once validated, these models can then be used to predict novel behaviors or to drive new experiments. Along this line, polymer-physics-based models have been instrumental in 3D genomics to better understand the structures and functions of chromosomes [24, 51] (see also the recent book edited by Giorgetti and Tiana [52] illustrating several existing approaches). In particular, in the context of TAD formation by loop extrusion process, polymer models have played a crucial role to quantitatively relate experiments to mechanisms involved in this complex process [31, 32]. Previously, generic, large-scale features like the formation of chromosome territories or the decay of the average contact frequency $P(s)$ between two loci as a function of their genomic distance $s\left(P(s) \propto s^{-1}\right.$ [5], Fig.1D) have been well captured by simple topologically-constrained self-avoiding polymers evolving in confined environment, the so-called crumpled or fractal polymer models [51, 53, 54]. Decoration of these models with specific interactions between monomers allowed to describe compartmentalization of chromatin into epigenomic compartments and TADs in higher eukaryotes [55-66]. In particular, the formation of mammalian TADs (with or without cornerpeaks, Fig. $1 \mathrm{~A}, \mathrm{C}$ ) might be captured by the partial collapse, the so-called theta- or coil-globule transition, of 1D chromatin domains eventually facilitated by point-like interactions between boundaries to model corner-peaks $[31,60]$. In these models, corner-peaks result from specific short-range interactions between CTCF-bound sites. However, such a molecular mechanism cannot physically explain why corner-peaks are observed only between sites with particular sense-antisense orientations [7, 32, 35] (Fig.2A). Models of loop extrusion recapitulate this intriguing observation along with the quantitative description of the average intra-TAD organization (Fig.1C) [31, 32]. In the following, we will review the three different types of existing polymer models for loop extrusion, depending on the main physical ingredient invoked to describe the translocation of extruders along chromatin. 


\subsection{Loop extrusion via translocating active motors}

Based on in vitro single-cell observations that condensin SMCs are able to processively compact DNA via an unknown ATP-dependent mechanism [37, 67], Alipour and Marko in 2012 [39] were the first to propose a theoretical framework to model the loop extrusion process by SMCs (Fig.2B). They modeled chromosome as a 1D lattice, neglecting the 3D polymeric nature of chromatin. A fixed number of loop extruding factors (LEFs) are initially loaded on the lattice and act as molecular motors by stochastically modifying the linear distance between the two LEF 'legs' leading to growing loops. LEFs can eventually unbind and re-attach at a different location, but cannot overlap or cross each other. Within this framework, Alipour and Marko showed that depending on the concentration of LEFs on the lattice and on the LEF processivity (ie the average genomic distance extruded by an isolated LEF during its lifetime on chromatin), two types of organization are observed: highly ordered stacked configurations for lower processivities and concentrations, and disordered configurations with smaller loops for larger processivities and concentrations. They suggested that mitotic condensation by condensin might result from the former regime with arrays of consecutive stacked loops stabilized by boundary elements (BEs) that halt the progression or favor the dissociation of LEFs.

To quantitatively interpret the current $\mathrm{Hi}-\mathrm{C}$ data of mammalian genome during interphase [7], Mirny's and Lieberman-Aiden's groups [31, 32], in 2015, developed physics-based models integrating a processive, unidirectional, loop extrusion process inspired by the seminal work of Alipour and Marko. Both approaches modeled chromosomes as 3D bead-and-string polymeric chains (600-1000 bp per monomer) which dynamically evolved under the action of LEFs (cohesin in interphase), modeled as rigid springs. LEFs bind at random position on chromatin, their two legs (i.e. the two edges of the spring) walking processively on chromatin in opposite directions, progressively extruding longer loops until they dissociate or reach a BE (CTCF sites with the correct orientation) where they stop (Fig.2C). Both models interpret phenomenologically the intra-TADs specificities (Fig.1) as the outcomes of this active translocation process: the average partial intra-TAD compaction and the corresponding scaling laws compared to inter-TAD properties (Fig.1C, D) result from the formation of dynamic loops by LEF only between regions belonging to the same TAD. Corner-peaks (Fig.1A) emerge when the processivity of LEFs is large enough for LEFs to reach both BEs at TAD borders before dissociation. The observation that corner-peaks represent contact mainly between convergent CTCF sites arises from the hypothesis that one LEF leg halts at BE only if the direction of translocation of the leg is opposite to the motif orientation, otherwise the leg may pass through the BE and continue its progression. Stripes (Fig.1B) may arise from preferential LEF loading closed to one BE [49]. In addition to BE positions and orientations, concentration and processivity of LEFs, as in Alipour and Marko's work, are the main physical ingredients controlling TAD folding (Fig.2D) with typical concentrations going from 1 LEF every $120 \mathrm{kbp}$ [32] to 1 LEF every $250 \mathrm{kbp}$ [31] and processivities between 120 and $240 \mathrm{kbp}$ in [32] or with higher values (>500 kbp) in [31]. Both models have shown remarkable (semi-) quantitative agreement with further perturbation experiments modifying BE locations or the levels of key components of the loop extrusion process [31,34]. For example, in the absence of BEs, models predict the disappearance of corner peaks and corresponding TADs but the maintenance of the scaling laws and even their extension beyond the former TAD boundaries, in agreement with CTCF knock-down experiments [43].

It is, however, important to note that these two models have significant differences, making it difficult to directly compare their parameters and predictions. Indeed, both use different 
null models for chromatin: Fudenberg et al [32] consider a semi-flexible chain with soft-core potential (weak excluded volume and possibility for polymer strands to cross each other) confined in a box with periodic boundary conditions, hence controlling the volumic density; Sanborn et al [31] adopt an isolated, self-avoiding, flexible chain with short-range, non-specific interactions between all monomers leading to a global collapse of the chain to mimic confinement. Another fundamental difference is the treatment of colliding LEFs and of LEF unbinding: in [32], LEFs follow a simple exclusion rule and may unbind stochastically chromatin at any time as in [39]; in [31], one of the two interfering LEFs dissociates randomly unless one LEF is already stalled at a BE and thus remains bound, otherwise random dissociation is very rare. Knowing that these two null models may have very different structural behaviors for $P(s)$ in the absence of LEFs $[51,54,68]$, one can easily understand that the optimal parameters for LEF concentrations and processivities are quite different between the two models, in particular, if they employ distinct collision and dissociation rules. For example, these differences may explain why Fudenberg et al correctly predicts average inter-TAD contact frequencies up to $1 \mathrm{Mbp}$ while Sanborn et al does not seem to be quantitative beyond the TAD-scale; or why Fudenberg et al observed generally symmetric stripes with corner-peaks as in Fig.1B, while Sanborn et al mainly predicted corner-peaks as in Fig.1A.

Building on their polymeric loop extrusion process by cohesin SMCs during interphase, Mirny's group also considered the role of condensin SMCs in the organization of prophase and mitotic chromosomes $[69,70]$. They showed that a high concentration of LEFs ( 1 LEF every $30 \mathrm{kbp}$ ) coupled to a high processivity ( $~ 800 \mathrm{kbp}$ ) leads to a dramatic compaction of chromosomes that naturally become elongated and are constituted of a dense array of consecutive loops of average size $100 \mathrm{kbp}$ without the need for BEs, recapitulating the initial results of Alipour and Marko [39] but with a more realistic and quantitative model. In particular, they observed that loop extrusion by condensin facilitates the disentanglement of entangled sister chromatids [70], in line with other works suggesting that loop extrusion may actively unknot polymeric chains [31, 71, 72]. A more recent study, coupling polymer modeling with loop extrusion and high-resolution $\mathrm{Hi}-\mathrm{C}$ experiments performed at different stages of mitosis, allowed to quantify the distinct roles of condensin I and II in mitotic chromosome formation in mammals [73]: condensin II may act first by creating a helical central scaffold with large consecutive loops ( $400 \mathrm{kbp}$ ), then, around this central axis, condensin I would compact the chromosome with shorter nested loops ( $80 \mathrm{kbp})$. The loop-extrusion-induced chromosome compaction by condensin is also believed to occur in bacteria, in which polymer models $[106,113]$ accounting for actively translocating LEFs loaded close to the origin of replication, can recapitulate the properties of bacterial genome folding observed in $\mathrm{Hi}-\mathrm{C}$ experiments [113].

\subsection{Loop extrusion via diffusing slip-links}

Despite numerous experimental studies in the last decade, the exact molecular mechanism of the formation of loops is not fully understood. Recent single-molecule studies illustrated the capacity of yeast condensins to actively extrude loops of naked dsDNA [40,41], demonstrating the potential translocating motor activity of SMCs. However, it is still unclear if cohesin is capable of such a movement, in particular on chromatin [74]. Rather, in vitro experiments on cohesin observed a random, diffusion-like dynamics [75-77,105].

To test whether the diffusion of LEFs may lead to TAD formation, Marenduzzo's group [78, 79, 107] developed a nonequilibrium model where LEFs bind and dissociate stochastically from chromatin, are free to diffuse as molecular slip-links [81] and are stabilized when reaching BEs (Fig.2E). Using first a 1D formulation and then generalizing it to integrate the 3D polymeric 
structure (chromatin as a confined semi-flexible standard 30nm-fiber [80] with $3 \mathrm{kbp}$ per monomer and LEFs as very rigid handcuffs), Brackley et al [78] showed that, in case of random loading of LEFs along the chain, the slip-links are distributed uniformly along the chromatin, which favors the formation of consecutive loop formation. As these loops compete with one another, they exhibit slow growth and the typical size of loops closed by the LEF decreases with the density of bound slip-links augments. This is incompatible with the formation of hundreds of kbps-long cohesin-CTCF loops observed for mammalian genomes. However, when LEF loading is limited to one specific site within a TAD, the model predicts the formation of nested loops. For the first-loaded slip-link, presence of all other bound diffusive LEFs on one of its sides and the total absence of LEFs on the other creates an osmotic pressure for the loop that may push the LEF towards its unoccupied side, i.e. towards the BEs. This osmotic ratchet leads to a dynamical, effective loop extrusion process and, contrary to random loading, generates a positive effect: more loaded slip-links result in larger loops. In particular, Brackley et al predicted that such positive osmotic effect may be observed if the 1D diffusion constant $D$ of slip-links is greater than $D \approx 0.025 \mu \mathrm{m}^{2} / \mathrm{s}$ which is well below the typical value $(D \sim 0.1-$ $0.25 \mu \mathrm{m}^{2} / \mathrm{s}$ ) measured in vitro [75-77]. They also observed that for a density of 1 LEF every 500 $\mathrm{kbp}$ and $\mathrm{D} \approx 5 \mathrm{kbp}^{2} / \mathrm{s}$, the formation of TADs with corner-peaks or stripes as large as $1.5 \mathrm{Mbp}$ is possible via this process. A similar model based on a discrete 3D lattice developed by Miermans \& Broderz [106] showed that the ratchet effect may also explain bacterial genome organization.

Concurrently to this work and following the same basic principle, a slightly different model was proposed by Yamamoto and Schiessel [82]. As in the work of Alipour and Marko [39], chromatin is treated as a $1 \mathrm{D}$ lattice, neglecting the 3D polymeric nature of the fiber. Each TAD is composed of a loading site at its center where cohesins may attach and unloading sites at domain borders where they dissociate. In addition to the loading of diffusive cohesin handcuffs (or dimers) as in [78], cohesin monomers (i.e. encompassing only one DNA strand, Fig.2E) may also associate at the same site and diffuse in 1D along the chain. These monomers also contribute to the osmotic pressure and thus reinforces the growth of loops entrapped by dimers, thus leading to loop extrusion. Hence, due to this extra contribution of monomers, the same osmotic pressure may be achieved with less loaded dimers.

While the mechanism of loop extrusion via diffusive slip-links is in line with in vitro experiments of the movement of single cohesin unit on chromatin [75-77] and shows qualitative agreement with $\mathrm{Hi}-\mathrm{C}$ data in mammals and in bacteria, it is still unclear whether such a mechanism is able to quantitatively predict the intra-TAD and inter-TAD scaling laws for the contact probability $P(S)$ (Fig.1C,D) as well as to predict TAD organization and loops depending on CTCF motif orientations or perturbation experiments in which CTCF or cohesin concentrations were modified [34]. Moreover, the osmotic ratchet effect requires the presence of only one loading site per TAD. While Chip-Seq profiles of Nipl (cohesin loader) exhibit peaks in TADs surrounding active genes at gene promoters, Nipl may have many secondary fixing sites or TADs with several active genes will have several Nipl peaks. However, a recent version of the diffusive slip-link model suggests that LEF loading at random positions may also lead to corner-peaked TADs [107].

\subsection{Loop extrusion via transcription-induced supercoiling}

As in the previous section, based on the lack of experimental proof for a direct translocation activity of cohesin SMCs, Stasiak's group suggested that transcription-induced negative supercoiling might be the motor for loop extrusion by cohesin [83, 84]. Previously, inspired by 
several studies suggesting that interphase chromosomes are torsionally stressed [85-87], the same group has developed a polymer model that accounts for possible supercoiling present within TADs [88]. They modeled chromatin as a confined (with periodic boundary conditions) bead-on-string self-avoiding polymer sensitive to torsional and bending stress, with TAD borders playing the role of anchors by maintaining a fixed stress within one domain. They showed that imposing a linking number difference of -2 turns per $100 \mathrm{kbp}$ leads to the formation of plectonemes that enhances the intra-TAD contact frequency with scaling laws compatible with experiments (Fig.1C), but the model failed to generate corner-peaks.

To better describe corner-peak TADs and the important role of cohesin and CTCF in TAD formation, Racko et al proposed (Fig.2F) that cohesins are loaded between the extremities of a transcribed region (a gene or an enhancer for example) within a TAD [83] and that a global negative torsional stress is produced by transcribing RNA polymerases [85], which can be relaxed by topoisomerase II B present at TAD borders. Transcription-induced stress leads to the formation of plectonemes between the active region and the cohesin SMC, transient stabilization of these plectonemes being made possible by the large hydrodynamic drag of the $\mathrm{SMC}$ anchoring the forming loop. This results in growing plectonemes that may push cohesin complexes towards BEs where the stress is eventually released by TOP2B. Cohesins reaching well-oriented BEs are then stabilized by interactions with bound CTCF. To test this mechanism, Racko et al developed a polymer model (hundreds of kbp in length with 400 bp per monomer) having a strong torsional persistence length that allows for the formation of plectonemes and limits the relaxation of the stress by internal twist; generation of negative supercoiling ( 5 to 10 turns) is performed by an active, rigid swivel positioned at the transcribed region; relaxation at BEs is allowed by passive swivels; cohesin SMCs being modeled as rigid handcuffs as in [78]. Movies extracted from molecular simulations of this model illustrated how the resulting flux of supercoiling (balance between creation at the transcribed region and relaxation at $\mathrm{BE}$ ) may push SMCs in TAD border directions. Though the transcription is essential for the TAD formation, the model suggested that once the extrusion is complete and cohesin complexes have reached both $\mathrm{BEs}$, the transcription is not required any more to maintain the TADs.

However, the lack of quantitative comparisons with $\mathrm{Hi}-\mathrm{C}$ data and of a precise impact analysis of model parameters makes it difficult to evaluate the validity of the proposed mechanism. While the enrichment of TOP2B at CTCF BEs is well documented [89, 90], the association between supercoiling domains observed in $[85,86]$ and TADs is still unclear. For example, a recent study showed that TOP2B activity at BEs is mainly transcription-independent and suggested that topological stress tends to occur at both sides of the BEs but not intra-TAD [90]. The main ingredient in the Stasiak's group model is the capacity of the polymer to absorb supercoiling stress by forming plectonemes instead of by twisting. This requires a strong torsional persistence length ( $\sim 100 \mathrm{~nm}$ corresponding to bare DNA) well beyond the actual experimental estimations (5 nm) [91] showing that short pieces of chromatin (10 kbp) can absorb many turns (40) before forming plectonemes [92]. Moreover, it is unclear how this mechanism of transcription-induced loop extrusion accounts for the cohesin-mediated formation of TADs with corner-peak in untranscribed or heterochromatic regions [44-46] and how it is consistent with the many perturbation experiments on BE locations and CTCF or cohesin levels. 


\section{Conclusion and Discussion}

Polymer models with various mechanisms and parameters have been proposed to model the generation of loops by extruding factors. All these models state that the formation of TADs and sub-structures like corner-peaks emerge from the dynamic translocation of SMC complexes. SMCs encompass two strands of chromatin and slide from the interior of the TAD to its boundary elements. However, the main physical ingredient leading directly or indirectly to this translocation differs between models: in [31, 32], cohesins are directly actively and unidirectionally translocated; in [78, 82], diffusive cohesins are translocated towards a preferential direction due to the osmotic pressure created by other cohesins diffusing on chromatin; in [83], transcription-induced supercoiling generates growing plectonemes that will push cohesin up to boundary elements. In addition, the stoichiometry of the extruding complexes (one or two SMC rings), the number of walking legs of an extruder, the possible self-interaction between motors and the molecular events leading to ATP-driven DNA capture and translocation are still unclear [74, 93-102]. Quantitative comparisons between these different modeling studies suffer from the use of different null polymer models to describe chromosomes, each assuming or using different properties to coarse-grain and simulate chromatin fibers. The development of a common theoretical framework based on a realistic null model [65] will certainly help to test the various existing hypotheses on loop extrusion under the same conditions. Another key point in these models is that, in order to explain the formation of corner-peaks only between convergent CTCF motifs, extruding factors are assumed to have differential interactions with these motifs depending on their orientations: either these sites act as a road-block or are passive in [31, 32], either they interact with extruders or reflect them in [78]. The molecular details of this asymmetric, orientationdependent effect still need to be investigated.

All this highlights the strong uncertainties existing about the correct molecular mechanisms responsible for loop extrusion. The most recent molecular experiments tend to suggest an active mechanism with only one walking leg [40] without steric hindrance between LEFs [41], but it was observed only for yeast condensin on dsDNA. Does it apply to cohesin whose motion seems more diffusive [75-77]? Does it happen on chromatin [100]? Future single-molecule studies on reconstituted chromatin in the presence of key controlled components (CTCF, TOP2B, etc.) measuring the dynamics but also the involved forces and torques will certainly help to resolve these ambiguities.

While other mechanisms driving chromosome organization like epigenomic-driven interactions, phase-separation or promoter-enhancer loops also start to be well characterized, it remains unclear how these different processes might interfere with loop extrusion. For example, Hi-C experiments on cohesin-depleted cells illustrated the loss of TAD organization replaced by a stronger compartmentalization between active and inactive compartments driven by epigenetics [45], indicating that loop extrusion process may destabilize compartment formation via active mixing [62]. Similarly, chromatin is also the template for many other motors like RNA polymerases during transcription, DNA polymerases during replication or translocases like Rad54 during DNA repair. It will be of course of interest to study the possible crosstalk between these processes. Along this line, recent experiments showed that transcription may influence cohesin localization $[108,109]$ or translocation $[77$, 103] but may also act as moving barriers against condensin progression [104]. Polymer physics-based models would certainly be very useful to probe such coupling and to suggest new exciting experiments. This will help to elucidate the contribution of loop extrusion in 
chromosome organization during interphase and mitosis and within different organisms, from bacteria to humans, in which SMC proteins play multiple roles, suggesting that the mechanisms of dynamic loop formation are generic and may be crucial to genome-folding regulation in all kingdoms of life.

\section{Acknowledgements}

We thank Cédric Vaillant, Maxime Tortora and Hossein Salari for careful reading of the manuscript. This work was supported by Agence National de la Recherche [ANR-15-CE120006-03, ANR-18-CE12-0006-03, ANR-18-CE45-0022-01] and ITMO Cancer [Plan Cancer 20142019, Biologie des Systèmes BIO2015-08].

\section{Author biography}

Surya K. Ghosh is Assistant Professor at SRM Institute of Science and Technology. His research interest is on the modeling of various biological phenomena: from the diffusion of single molecules to the organization of long polymers.

Daniel Jost is Research Associate at CNRS and head of the 'Physical Biology of Chromatin' group at Ecole Normale Supérieure de Lyon. He is an expert in biophysical modeling of living systems. His group mainly focuses on epigenetic regulation and chromosome organization.

\section{Figures}

FIG. 1: (A, B) Pieces of Hi-C maps of chromosome 4 (A) and 14 (B) for the human cell line GM12878 [7] illustrating the presence of corner-peaks (arrows in A) or of stripes (arrows in B) at TAD boundaries. For visual inspection, corresponding Chip-seq signals for CTCF and cohesin, as well as the position and orientation (green: sense, red: antisense) of CTCF motifs were added. Panels A and B were built using the webtool Juicebox [50]. (C, D) Average contact frequency $P(s)$ between two loci separated by a genomic distance s: intra-TAD frequencies for TADs of different sizes in presence or not of corner-peaks $(C)$ and inter-TAD frequency (D). Dashed lines represent scaling laws $P(s) \propto s^{-\nu}$.

FIG. 2: Different models for loop extrusion. (A) If the genomic distance between two oriented motifs is larger than the torsional persistence length of chromatin, no preferential orientation of interaction will be observed for the pair. (B) Nasmyth \& Riggs model for mitotic condensation by condensins resembling a loop extrusion process: condensins bind to chromatin and form an array of consecutive loops and an axial core. (C) Loop extrusion by active, unidirectional translocating motors: LEFs are loaded to and unloaded from chromatin randomly, the two 'legs' of a LEF walk in opposite directions, one leg is halted when reaching a CTCF binding motif oriented opposite to its translocation direction. (D) Representation of different types of local organization as a function of LEF concentration and processivity. For low concentrations and processivities, sparse arrays of contiguous loops of small sizes dominate. As the concentration is increased, arrays become denser; as the processivity is increased, loops become larger, with eventually, the presence of stacked loops. (E) Loop extrusion by diffusive slip-links: SMCs are loaded as dimers or monomers at specific sites and diffuse along the chromatin, dimers interact with boundary elements oriented opposite to their motion while being reflected by $B E$ in the other direction. (F) Loop extrusion by transcription-induced supercoiling: LEFs bind to a specific - transcribed - region, supercoiling 
generated by transcription leads to plectoneme formation that push LEFs which are stabilized at BE.

\section{References}

[1] Allis, C., Jenuwein, T. \& Reinberg, D. Epigenetics (Cold Spring Harbor Laboratory Press, 2007).

[2] de Wit, E. \& de Laat, W. A decade of 3C technologies: insights into nuclear organization. Genes \& Development 26, 11-24 (2012).

[3] Cremer, C., Szczurek, A., Schock, F. et al. Super-resolution microscopy approaches to nuclear nanostructure imaging. Methods 123, 11-32 (2017).

[4] Bonev, B. \& Cavalli, G. Organization and function of the $3 \mathrm{~d}$ genome. Nature Reviews Genetics 17, 661-678 (2016).

[5] Lieberman-Aiden, E., van Berkum, N. L., Williams, L. et al. Comprehensive mapping of longrange interactions reveals folding principles of the human genome. Science 326, 289-293 (2009).

[6] Sexton, T., Yaffe, E., Kenigsberg, E. et al. Three-dimensional folding and functional organization principles of the drosophila genome. Cell 148, 458-472 (2012).

[7] Rao, S. S., Huntley, M. H., Durand, N. C et al. A 3D map of the human genome at kilobase resolution reveals principles of chromatin looping. Cell 159, 1665-1680 (2014).

[8] Cremer, T., Cremer, M., Hübner, B. et al. The 4D nucleome: Evidence for a dynamic nuclear landscape based on co-aligned active and inactive nuclear compartments. FEBS Letters 589, 2931-2943 (2015).

[9] Gerlich, D., Beaudouin, J., Kalbfuss, B et al. Global chromosome positions are transmitted through mitosis in mammalian cells. Cell 112, $751-764$ (2003).

[10] Bolzer, A., Kreth, G., Solovei, I. et al. Three-dimensional maps of all chromosomes in human male fibroblast nuclei and prometaphase rosettes. PLoS Biology 3, e157 (2005).

[11] Mattout, A., Cabianca, D. S. \& Gasser, S. M. Chromatin states and nuclear organization in development - a view from the nuclear lamina. Genome Biology 16, 174 (2015).

[12] Falk, M., Feodorova, Y., Naumova, N. et al. Heterochromatin drives compartmentalization of inverted and conventional nuclei. Nature, in press (2019).

[13] Bagadia, M., Singh, A. \& Sandhu, K. S. Three-dimensional organization of genome might have guided the dynamics of gene order evolution in eukaryotes. Genome Biology and Evolution 8, 946-954 (2016).

[14] Lupianez, D. G., Kraft, K., Heinrich, V. et al. Disruptions of topological chromatin domains cause pathogenic rewiring of gene-enhancer interactions. Cell 161, 1012-1025 (2015).

[15] Quintero-Cadena, P. \& Sternberg, P. W. Enhancer sharing promotes neighborhoods of transcriptional regulation across eukaryotes. G3: Genes, Genomes, Genetics 6, 4167-4174 (2016).

[16] Franke, M., Ibrahim, D. M., Andrey, G. et al. Formation of new chromatin domains determines pathogenicity of genomic duplications. Nature 538, 265-269 (2016).

[17] Corces, M. R. \& Corces, V. G. The three-dimensional cancer genome. Current opinion in genetics \& development 36, 1-7 (2016).

[18] Ruiz-Velasco, M. \& Zaugg, J. B. Structure meets function: how chromatin organisation conveys functionality. Current Opinion in Systems Biology 1, 129-136 (2017).

[19] Kaiser, V. B. \& Semple, C. A. When tads go bad: chromatin structure and nuclear organisation in human disease. F1000Research 6 (2017). 
[20] Hao, N., Shearwin, K. E. \& Dood, I. B. Positive and negative control of enhancer-promoter interactions by other DNA loops generates specificity and tunability. Cell Reports 26, 24192433 (2019).

[21] Rodriguez-Carballo, E., Lopez-Delisle, L., Yakushiji-Kaminatsui, $\mathrm{N}$ et al. Impact of genome architecture upon the functional activation and repression of hox regulatory landscapes. bioRxiv 587301 (2019).

[22] Williamson, I., Kane, L., Devenney, P. S. et al. Developmentally regulated Shh expression is robust to tad perturbations. bioRxiv 609941 (2019).

[23] Ghavi-Helm, Y., Jankowski, A., Meiers, S. et al. Highly rearranged chromosomes reveal uncoupling between genome topology and gene expression. Nature Genetics, in press (2019). [24] Jost, D., Vaillant, C. \& Meister, P. Coupling 1D modifications and 3D nuclear organization: data, models and function. Curr Opin Cell Biol 44, 20-27 (2017).

[25] Canzio, D. et al. A conformational switch in HP1 releases auto-inhibition to drive heterochromatin assembly. Nature 496, 377-381 (2013).

[26] Isono, K., Liao, M., Naber, N. et al. Sam domain polymerization links subnuclear clustering of PRC1 to gene silencing. Dev Cell 26, 565-577 (2013).

[27] Machida, S., Takizawa, Y., Ishimaru, M. et al. Structural basis of heterochromatin formation by human HP1. Molecular Cell 69, 385-397 (2018).

[28] Strom, A. R., Emelyanov, A. V., Mir, M. et al. Phase separation drives heterochromatin domain formation. Nature 547, 241-245 (2017).

[29] Larson, A. G., Elnatan, D., Keenen, M. M. et al. Liquid droplet formation by HP1 $\alpha$ suggests a role for phase separation in heterochromatin. Nature 547, 236-240 (2017).

[30] Shin, Y., Chang, I.-C., Lee, D. S. et al. Liquid nuclear condensates mechanically sense and restructure the genome. Cell 175, 1481-1491 (2018).

[31] Sanborn, A. L., Rao, S. S. P., Huang, S.-C. et al. Chromatin extrusion explains key features of loop and domain formation in wild-type and engineered genomes. Proceedings of the National Academy of Sciences USA 112, E6456-E6465 (2015).

[32] Fudenberg, G., Imakaev, M., Lu, C. et al. Formation of Chromosomal Domains by Loop Extrusion. Cell Reports 15, 2038-2049 (2016).

[33] Dekker, J. \& Mirny, L. The 3D Genome as Moderator of Chromosomal Communication. Cell 164, 1110-1121 (2016).

[34] Fudenberg, G., Abdennur, N., Imakaev et al. Emerging evidence of chromosome folding by loop extrusion. Cold Spring Harbor Symposia on Quantitative Biology 82, 42-55 (2017).

[35] Dowen, J. M., Fan, Z. P., Hnisz, D. et al. Control of cell identity genes occurs in insulated neighborhoods in mammalian chromosomes. Cell 159, 374-387 (2014).

[36] Vietri Rudan, M., Barrington, C., Henderson, S et al. Comparative Hi-C reveals that CTCF underlies evolution of chromosomal domain architecture. Cell Rep 10, 1297-1309 (2015).

[37] Kimura, K., Rybenkov, V. V., Crisona, N. J. et al. 13s condensin actively reconfigures dna by introducing global positive writhe: implications for chromosome condensation. Cell (1999). [38] Nasmyth, K. Disseminating the genome: Joining, resolving, and separating sister chromatids during mitosis and meiosis. Annual Review of Genetics 35, 673-745 (2001).

[39] Alipour, E. \& Marko, J. F. Self-organization of domain structures by DNA-loop-extruding enzymes. Nucleic Acids Research 40, 11202-11212 (2012).

[40] Ganji, M., Shaltiel, I. A., Bisht, S. et al. Real-time imaging of DNA loop extrusion by condensin. Science 360, 102-105 (2018).

[41] Kim, E., Kerssemakers, J., Shaltiel, I. A. et al. DNA-loop extruding condensin complexes can traverse one another. bioRxiv 682864 (2019). 
[42] Guo, Y., Xu, Q., Canzio, D. et al. Crispr inversion of CTCF sites alters genome topology and enhancer/promoter function. Cell 162, 900-910 (2015).

[43] Nora, E. P., Goloborodko, A., Valton, A.-L. et al. Targeted degradation of CTCF decouples local insulation of chromosome domains from genomic compartmentalization. Cell 169, 930944 (2017).

[44] Wutz, G., Várnai, C., Nagasaka, K. et al. Topologically associating domains and chromatin loops depend on cohesin and are regulated by CTCF, Wapl, and Pds5 proteins. EMBO Journal 36, 3573-3599 (2017).

[45] Schwarzer, W., Abdennur, N., Goloborodko, A. et al. Two independent modes of chromosome organization are revealed by cohesin removal. Nature 551, 51-56 (2017).

[46] Rao, S. S., Huang, S.-C., St Hilaire, B. G. et al. Cohesin loss eliminates all loop domains. Cell 171, 305-320 (2017).

[47] Gassler, J., Brandão, H. B., Imakaev, M. et al. A mechanism of cohesin-dependent loop extrusion organizes zygotic genome architecture. EMBO Journal 36, 3600-3618 (2017).

[48] Haarhuis, J. H., van der Weide, R. H., Blomen, V. A. et al. The cohesin release factor Wapl restricts chromatin loop extension. Cell 169, 693-707 (2017).

[49] Vian, L., Pekowska, A., Rao, S. S. et al. The energetics and physiological impact of cohesin extrusion. Cell 173, 1165-1178 (2018).

[50] Durand, N. C., Robinson, J. T., Shamim, M. S. et al. Juicebox provides a visualization system for Hi-C contact maps with unlimited zoom. Cell Systems 3, 99-101 (2016).

[51] Mirny, L. A. The fractal globule as a model of chromatin architecture in the cell. Chromosome Res 19, 37-51 (2011).

[52] Tiana, G. \& Giorgetti, L. (eds.) Modeling the 3D Conformation of Genomes (CRC Press, 2019).

[53] Rosa, A. \& Everaers, R. Structure and dynamics of interphase chromosomes. PLoS Comput. Biol. 4, e1000153 (2008).

[54] Halverson, J. D., Smrek, J., Kremer, K. et al. From a melt of rings to chromosome territories: the role of topological constraints in genome folding. Rep Prog Phys 77, 022601 (2014).

[55] Barbieri, M., Chotalia, M., Fraser, J. et al. Complexity of chromatin folding is captured by the strings and binders switch model. Proc Natl Acad Sci USA 109, 16173-16178 (2012).

[56] Ganai, N., Sengupta, S. \& Menon, G. I. Chromosome positioning from activity-based segregation. Nucleic Acids Res 42, 4145-4159 (2014).

[57] Jost, D., Carrivain, P., Cavalli, G. et al. Modeling epigenome folding: formation and dynamics of topologically associated chromatin domains. Nucleic Acids Res 42, 9553-9561 (2014).

[58] Brackley, C. A., Johnson, J., Kelly, S. et al. Simulated binding of transcription factors to active and inactive regions folds human chromosomes into loops, rosettes and topological domains. Nucleic Acids Res 44, 3503-3512 (2016).

[59] Olarte-Plata, J. D., Haddad, N., Vaillant, C. et al. The folding landscape of the epigenome. Phys Biol 13, 026001 (2016).

[60] Di Pierro, M., Cheng, R. R., Aiden, E. L. et al. De novo prediction of human chromosome structures: Epigenetic marking patterns encode genome architecture. Proc Natl Acad Sci USA 114, 12126-12131 (2017).

[61] Buckle, A., Brackley, C. A., Boyle, S. et al. Polymer simulations of heteromorphic chromatin predict the 3D folding of complex genomic loci. Molecular Cell 72, 786-797 (2018). 
[62] Nuebler, J., Fudenberg, G., Imakaev, M. et al. Chromatin organization by an interplay of loop extrusion and compartmental segregation. Proc Natl Acad Sci USA 115, E6697-E6706 (2018).

[63] Shi, G., Liu, L., Hyeon, C. et al. Interphase human chromosome exhibits out of equilibrium glassy dynamics. Nature Communications 9, 3161 (2018).

[64] Bianco, S., Lupianez, D. G., Chiariello, A. M. et al. Polymer physics predicts the effects of structural variants on chromatin architecture. Nature Genetics 50, 662-667 (2018).

[65] Ghosh, S. K. \& Jost, D. How epigenome drives chromatin folding and dynamics, insights from efficient coarse-grained models of chromosomes. PLoS Computational Biology 14, e1006159 (2018).

[66] Lesage, A., Dahirel, V., Victor, J.-M. et al. Polymer coil-globule phase transition is a universal folding principle of drosophila epigenetic domains. Epigenetics \& Chromatin 12, 28 (2019).

[67] Strick, T., Kawaguchi, T. \& Hirano, T. Real-time detection of single-molecule DNA compaction by condensin I. Current Biology 14, 874-880 (2004).

[68] Doi, M. \& Edwards, S. F. The Theory of Polymer Dynamics (Oxford University Press, New York, 1986).

[69] Goloborodko, A., Imakaev, M. V., Marko, J. F. et al. Compaction and segregation of sister chromatids via active loop extrusion. Elife 5 (2016).

[70] Goloborodko, A., Marko, J. F. \& Mirny, L. A. Chromosome compaction by active loop extrusion. Biophys J 110, 2162-2168 (2016).

[71] Racko, D., Benedetti, F., Goundaroulis, D. et al. Chromatin loop extrusion and chromatin unknotting. Polymers 10, 1126 (2018).

[72] Orlandini, E., Marenduzzo, D. \& Michieletto, D. Synergy of topoisomerase and structuralmaintenance-of-chromosomes proteins creates a universal pathway to simplify genome topology. Proc Natl Acad Sci USA 116, 8149-8154 (2019).

[73] Gibcus, J. H., Samejima, K.; Goloborodko, A. et al. A pathway for mitotic chromosome formation. Science 359 (2018).

[74] Uhlmann, F. SMC complexes: from DNA to chromosomes. Nature Reviews Molecular Cell Biology 17, 399-412 (2016).

[75] Stigler, J., Camdere, G.O., Koshland, D.E. et al. Single-Molecule Imaging Reveals a Collapsed Conformational State for DNA-Bound Cohesin. Cell reports 15, 988-998 (2016).

[76] Kanke, M., Tahara, E., Huis in't Veld, P. J. et al. Cohesin acetylation and Wapl-Pds5 oppositely regulate translocation of cohesin along DNA. The EMBO Journal 35, 2686-2698 (2016).

[77] Davidson, I. F., Goetz, D., Zaczek, M. P. et al. Rapid movement and transcriptional relocalization of human cohesin on DNA. The EMBO Journal 35, 2671-2685 (2016).

[78] Brackley, C. A., Johnson, J., Michieletto, D. et al. Nonequilibrium chromosome looping via molecular slip links. Phys. Rev. Lett. 119, 138101 (2017).

[79] Brackley, C. A., Johnson, J., Michieletto, D. et al. Extrusion without a motor: a new take on the loop extrusion model of genome organization. Nucleus 9, 95-103 (2018).

[80] Munkel, C. \& Langowski, J. Chromosome structure predicted by a polymer model. Phys Rev E 57, 5888-5896 (1998).

[81] Ball, R., Doi, M., Edwards, S. et al. Elasticity of entangled networks. Polymer 22, 1010 1018 (1981).

[82] Yamamoto, T. \& Schiessel, H. Osmotic mechanism of the loop extrusion process. Phys. Rev. E 96, 030402 (2017). 
[83] Racko, D., Benedetti, F., Dorier, J. et al. Transcription-induced supercoiling as the driving force of chromatin loop extrusion during formation of TADs in interphase chromosomes. Nucleic Acids Research 46, 1648-1660 (2017).

[84] Racko, D., Benedetti, F., Dorier, J. et al. Are TADs supercoiled? Nucleic Acids Research 47, 521-532 (2018).

[85] Naughton, C., Avlonitis, N., Corless, S. et al. Transcription forms and remodels supercoiling domains unfolding large-scale chromatin structures. Nature Structural \& Molecular Biology 20, 387 (2013).

[86] Kouzine, F., Gupta, A., Baranello, L. et al. Transcription-dependent dynamic supercoiling is a short-range genomic force. Nature Structural \& Molecular Biology 20, 396 (2013).

[87] Bjorkegren, C. \& Baranello, L. DNA supercoiling, topoisomerases, and cohesin: Partners in regulating chromatin architecture? International Journal of Molecular Sciences 19 (2018).

[88] Benedetti, F., Dorier, J., Burnier, Y. et al, A. Models that include supercoiling of topological domains reproduce several known features of interphase chromosomes. Nucleic Acids Res 42, 2848-2855 (2014).

[89] Uuskula-Reimand, L., Hou, H., Samavarchi-Tehrani, P. et al. Topoisomerase ii beta interacts with cohesin and CTCF at topological domain borders. Genome Biology 17, 182 (2016).

[90] Canela, A., Maman, Y., Jung, S. et al. Genome organization drives chromosome fragility. Cell 170, 507-521 (2017).

[91] Bancaud, A., Conde e Silva, N., Barbi, M. et al. Structural plasticity of single chromatin fibers revealed by torsional manipulation. Nature Structural \& Molecular Biology 13, 444-450 (2006).

[92] Celedon, A., Nodelman, I. M., Wildt, B. et al. Magnetic tweezers measurement of single molecule torque. Nano Letters 9, 1720-1725 (2009).

[93] Gruber, S. Shaping chromosomes by DNA capture and release: gating the SMC rings. Current Opinion in Cell Biology 46, 87-93 (2017).

[94] Lawrimore, J., Friedman, B., Doshi, A. et al. Rotostep: A chromosome dynamics simulator reveals mechanisms of loop extrusion. Cold Spring Harb Symp Quant Biol 22, 033696 (2017).

[95] Sakai, Y., Mochizuki, A., Kinoshita, K. et al. Modeling the functions of condensin in chromosome shaping and segregation. PLoS Comput. Biol. 14, e1006152 (2018).

[96] Banigan, E. J. \& Mirny, L. A. Limits of chromosome compaction by loop-extruding motors. bioRxiv 476424 (2018).

[97] Murayama, Y., Samora, C. P., Kurokawa, Y. et al. Establishment of DNA-DNA interactions by the cohesin ring. Cell 172, 465-477 (2018).

[98] Marko, J. F., De Los Rios, P., Barducci, A. et al. DNA-segment-capture model for loop extrusion by structural maintenance of chromosome (SMC) protein complexes. Nucleic Acids Research gkz497 (2019).

[99] Hassler, M., Shaltiel, I. A., Kschonsak, M. et al. Structural basis of an asymmetric condensin ATPase cycle. Molecular Cell 74, 1175-1188 (2019).

[100] Kong, M., Cutts, E., Pan, D. et al. Human condensin I and II drive extensive ATPdependent compaction of nucleosome-bound DNA. bioRxiv 683540 (2019).

[101] Srinivasan, M., Scheinost, J. C., Petela, N. J. et al. The Cohesin Ring Uses Its Hinge to Organize DNA Using Non-topological as well as Topological Mechanisms. Cell 173, 1508-1519 (2018).

[102] Chapard, C., Jones, R., Oepen, T. et al. Sister DNA Entrapment between Juxtaposed Smc Heads and Kleisin of the Cohesin Complex. Mol Cell, in press (2019). 
[103] Borrie, M. S., Campor, J. S., Joshi, H. et al. R. Binding, sliding, and function of cohesin during transcriptional activation. Proc Natl Acad Sci USA 114, E1062-1071 (2017).

[104] Brandao, H. B., Wang, X., Paul, P. et al. RNA polymerases as moving barriers to condensin loop extrusion. bioRxiv 604280 (2019).

[105] Terakawa, T., Bisht, S., Eeftens, J.M. et al. The condensin complex is a mechanochemical motor that translocates along DNA. Science 358, 672-676 (2017).

[106] Miermans, C.A. \& Broedersz, C.P. Bacterial chromosome organization by collective dynamics of SMC condensins. J. R. Soc. Interface 15, 20180495 (2018)

[107] Pereira, M.C.F., Brackley, C.A., Michieletto, D. et al. Complementary chromosome folding by transcription factors and cohesin. bioRxiv 305359 (2018).

[108] Ocampo-Hafalla, M.T. \& Uhlmann, F. Cohesin loading and sliding. J. Cell Sci. 124, 685691 (2011).

[109] Busslinger, G.A., Stocsits, R.R., van der Lelij, P, et al. Cohesin is positioned in mammalian genomes by transcription, CTCF and Wapl. Nature 544, 503-507 (2017).

[110] Riggs, A.D. DNA methylation and late replication probably aid cell memory, and type I DNA reeling could aid chromosome folding and enhancer function. Phil. Trans. R. Soc. Lond. B 326, 285-297 (1990).

[111] Eeftens, J.M, Bisht, S., Kerssemakers, J. et al. Real-time detection of condensin-driven DNA compaction reveals a multistep binding mechanism. EMBO J. 36, 3448-3457 (2017).

[112] Schalbetter, S.A., Fudenberg, G., Baxter, J, et al. Principles of meiotic chromosome assembly. bioRxiv 442038 (2019).

[113] Wang, X., Brandao, H.B., Le, T.B.K, et al. Bacillus subtilis SMC complexes juxtapose chromosome arms as they travel from origin to terminus. Science 355, 524-527 (2017). 